\title{
Amplicon-based skin microbiome profiles collected by tape stripping with different adhesive film dressings: a comparative study
}

Kazuhiro Ogai ${ }^{1,2^{*}}$ (D, Kana Shibata ${ }^{3 \dagger}$, Natsuki Takahashi ${ }^{3+}$, Kohei Ogura ${ }^{4}$, Shigefumi Okamoto ${ }^{3}$ and Junko Sugama ${ }^{2,4^{*}}$

\begin{abstract}
Background: Medical film dressings have been used to obtain skin microbiota for skin microbiome studies, although their adhesive force may be so strong that the skin could be injured when applied to those who have fragile skin, such as older people. Several products with less adhesive force are available, although their applicability for skin microbiome studies remains unknown. This study aimed to test whether the dressings with less adhesive force could be used for amplicon-based skin microbiome studies. A set of three different film dressings, with acrylic, urethane, or silicone adhesive, was applied to the back skin of nine healthy young participants. The copy number of the $16 \mathrm{~S}$ ribosomal RNA (rRNA) gene, microbial compositions, and alpha and beta diversity indices were analyzed by amplicon analysis of the $16 \mathrm{~S}$ rRNA gene using next-generation sequencing and were compared among the three film dressings.

Results: The dressing with acrylic adhesive yielded the highest copy number of 165 rRNA genes, followed by that with urethane adhesive. The silicone-adhesive dressing yielded a significantly lower copy number of the 16S rRNA gene. The microbial composition of skin microbiota was similar among the three film dressings, although significant differences in the relative abundance of Pseudomonas species and alpha diversity indices were found in the silicone-adhesive dressing. The Bray-Curtis dissimilarity was significantly higher between the acrylic- and siliconeadhesive dressings than between the acrylic- and urethane-adhesive dressings. No adverse effects related to tape stripping were observed for any of the film dressings.

(Continued on next page)
\end{abstract}

\footnotetext{
*Correspondence: kazuhiro@staff.kanazawa-u.ac.jp;

junkosgm@mhs.mp.kanazawa-u.ac.jp

${ }^{\dagger}$ Kana Shibata and Natsuki Takahashi contributed equally to this work.

${ }^{1}$ Al Hospital/Macro Signal Dynamics Research and Development Center,

Institute of Medical, Pharmaceutical and Health Sciences, Kanazawa

University, 5-11-80 Kodatsuno, Kanazawa, Ishikawa 9200942, Japan

2Department of Nursing, Faculty of Health Sciences, Institute of Medical,

Pharmaceutical and Health Sciences, Kanazawa University, 5-11-80

Kodatsuno, Kanazawa, Ishikawa 9200942, Japan

Full list of author information is available at the end of the article
}

(C) The Author(s). 2021 Open Access This article is licensed under a Creative Commons Attribution 4.0 International License, which permits use, sharing, adaptation, distribution and reproduction in any medium or format, as long as you give appropriate credit to the original author(s) and the source, provide a link to the Creative Commons licence, and indicate if changes were made. The images or other third party material in this article are included in the article's Creative Commons licence, unless indicated otherwise in a credit line to the material. If material is not included in the article's Creative Commons licence and your intended use is not permitted by statutory regulation or exceeds the permitted use, you will need to obtain permission directly from the copyright holder. To view a copy of this licence, visit http://creativecommons.org/licenses/by/4.0/ The Creative Commons Public Domain Dedication waiver (http://creativecommons.org/publicdomain/zero/1.0/) applies to the data made available in this article, unless otherwise stated in a credit line to the data. 
(Continued from previous page)

Conclusion: We recommend dressings with acrylic or urethane adhesive for amplicon-based skin microbiome studies. An acrylic adhesive has an advantage in the yield of skin microbiota, and a urethane adhesive should be chosen when applied to fragile skin. The adhesive force of the dressing with silicone adhesive was too weak to be used for collecting skin microbiota.

Keywords: Skin, Microbiome, Tape-stripping, Adhesive, $16 \mathrm{~S}$ rRNA, Next generation sequencing

\section{Background}

As the largest organ, the skin serves as the physical barrier of our body, not only by protecting from external insults but also by retaining water inside the body [1]. Skin immunity also forms a barrier against the invasion of pathogenic bacteria or foreign substances [2]. In contrast, systemic diseases, such as diabetes, skin diseases, such as atopic dermatitis and psoriasis, or merely aging can affect the skin's integrity, leading to a barrier breakdown [3].

The relationship between host and skin bacteria has long been discussed in terms of pathogenicity [4], immunological interaction $[5,6]$, and physiological barrier function [7]. Studies on cutaneous bacteria and its relationship with the host have been accelerated by the development of high-throughput sequencing techniques, namely, next-generation sequencing (NGS) analysis of the $16 \mathrm{~S}$ ribosomal RNA (rRNA) $[8,9]$. However, as the skin harbors more diverse and less abundant microbiota than the other parts of our body (e.g., gut), a more careful design of skin microbiome studies is required [10].

The factors that affect the results of skin microbiome studies include the method used to obtain the samples of skin bacteria (e.g., skin biopsy, swabbing, skin scraping, cup scrubbing, or tape stripping), the DNA extraction method, the region of the $16 \mathrm{~S}$ rRNA gene to be sequenced, and the software and database employed [10]. Of them, the sampling method needs to be determined before performing a series of studies, as this cannot be changed after sampling.

Although the swabbing method has been widely used to collect skin microbiota for microbiome studies, the tape-stripping method has also been used due to its stability of collection maneuver, better microbiome coverage, and ease of use [11-13]. As skin microbiota exists across the layers of the stratum corneum, and the microbial composition can be different according to its depth $[14,15]$, the tape-stripping method is also preferred when focusing on the inner microbiome in the stratum corneum.

Several skin microbiome studies have successfully used medical-grade film dressings (thin, sterile, air-permeable medical tapes) for the tape-stripping method because they have been validated to obtain comparable results with traditional swabbing methods for collecting skin microbiome [12, 13]. However, one problem that is encountered with the use of film dressings is that its stickiness may cause skin injuries, especially when sampling from infants or older people who have fragile skin. The skin of older people is very thin, making it extremely fragile against even minimal invasion, including peeling off the tape [16-18]. Even with medical-grade film dressings, skin injuries may occur, called medical adhesive-related skin injuries (MARSI) [19]. To prevent MARSI, film dressings with less sticky adhesives than commonly-used acrylic adhesive, such as a silicone or urethane gel glue, have been introduced in clinical settings $[16,17,20,21]$. When the tape-stripping method is used for skin microbiome analysis on those who have fragile skin, researchers need to choose less invasive adhesives, such as urethane or silicone adhesive, without sacrificing the yield and fidelity to the composition of the skin microbiome. Therefore, we first need to clarify whether there are differences in skin microbiome results when different adhesives are used.

The aim of this study was to compare the microbiome data obtained from three different film dressings with different adhesives (acrylic, urethane, or silicone) to test the applicability of these film dressings for ampliconbased skin microbiome studies.

\section{Results}

\section{Adverse effects of tape stripping}

No adverse effects during tape stripping (e.g., pain, redness, irritation, skin breakdown, etc.) were observed throughout this study.

\section{S rRNA gene copy number}

Figure 1 shows the copy number of the 16S rRNA genes of each type of film dressing. The film dressing with silicone adhesive yielded significantly fewer copies of the 16S rRNA gene than that with acrylic adhesive $(P<$ $0.001)$. There were no significant differences in the copy number of the 16S rRNA gene between the acrylic and urethane adhesives $(P=0.086)$ and between the urethane and silicone adhesives $(P=0.086)$.

\section{Bacterial composition}

The bacterial composition obtained from the three different film dressings is summarized in Fig. 2 (with 


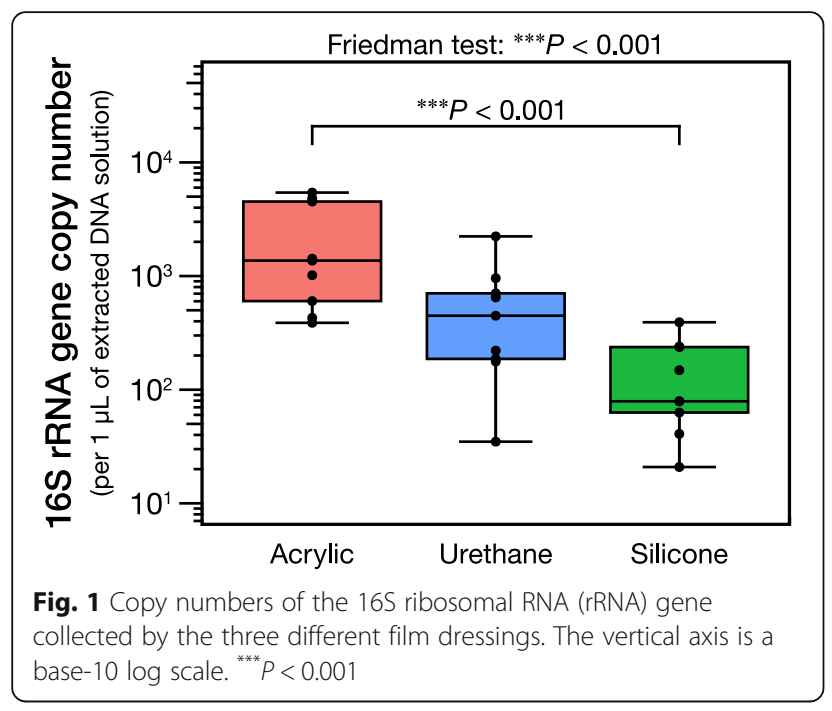

Additional file 1: Figure S1 for each participant). All three dressings obtained the same top five genera (Cutibacterium species [spp.], Staphylococcus spp., Pseudomonas spp., Corynebacterium spp., and Enhydrobacter spp.), all of which have been reported as skin commensals $[22,23]$. However, the relative abundances of these bacteria were apparently different between the silicone adhesive and the other two adhesives.

Detailed comparisons of the top five genera are shown in Fig. 3, and a top 20 comparison is shown in Table 1. Of the top five genera, Pseudomonas spp. showed a significant difference between acrylic and silicone adhesives $(P=0.013)$. The other top five bacteria did not show any significant differences between the three film dressings, although the silicone adhesive yielded slightly different values compared with the other two adhesives. The results of the amplicon sequence variants (ASVs)-level comparison, in which ASVs that showed more than 0.1\% of average relative abundance are listed, are shown in Additional file 2: Table S1. Similar to the genus-level comparison, the silicone adhesive showed significantly different value in one ASV (9th rank ASV).

\section{Beta diversity}

We performed a principal coordinate analysis plot based on Bray-Curtis dissimilarity (Fig. 4a). There were no statistically significant differences in the pattern of beta diversity, as evaluated by the pairwise permutational multivariate analysis of variance (PERMANOVA) analysis (Table 2). The Bray-Curtis dissimilarity itself showed a significantly higher value between the acrylicand silicone-adhesive film dressings than between the acrylic- and urethane-adhesive film dressings $(P=0.028$; Fig. 4b).

\section{Alpha diversity}

The alpha diversity indices (observed ASVs, phylogenetic diversity, and Shannon index) are shown in Fig. 5. The silicone-adhesive film dressing showed significantly higher alpha diversity indices than the acrylic or urethane adhesive film dressing, except for observed ASVs.

\section{Discussion}

We tested film dressings with three different adhesives (acrylic, urethane, and silicone) as a means of collecting skin microbiota samples. We found that acrylic and urethane adhesives obtained similar results, and the use of silicone-adhesive film dressing led to biased outcomes for amplicon-based skin microbiome analyses.
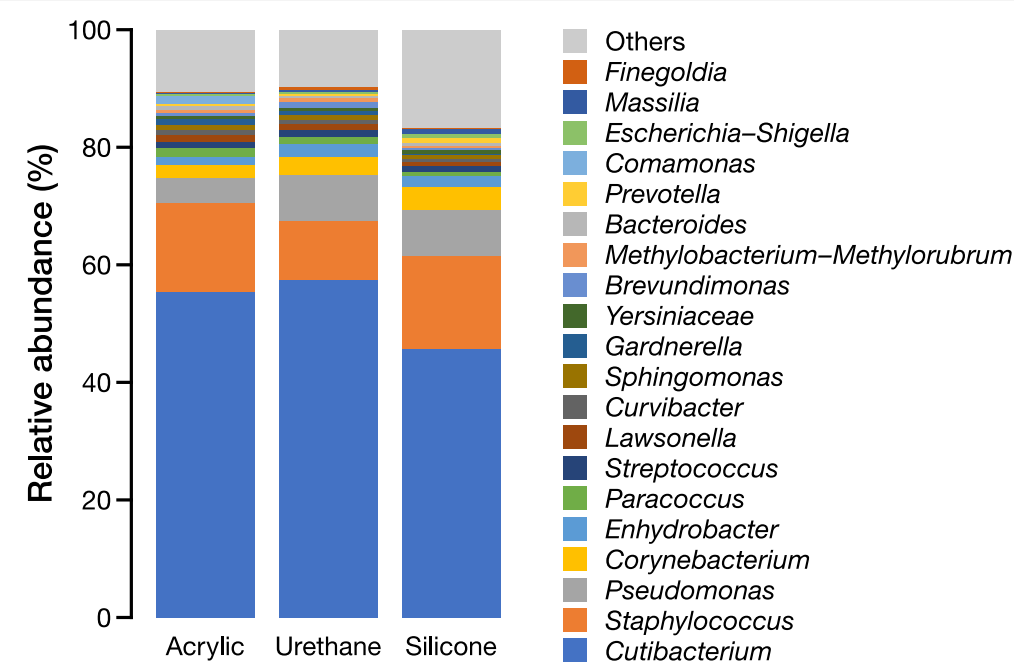

Fig. 2 Average relative abundance of the top 20 skin microbiota collected by the three different film dressings (acrylic, urethane, and silicone adhesives) 


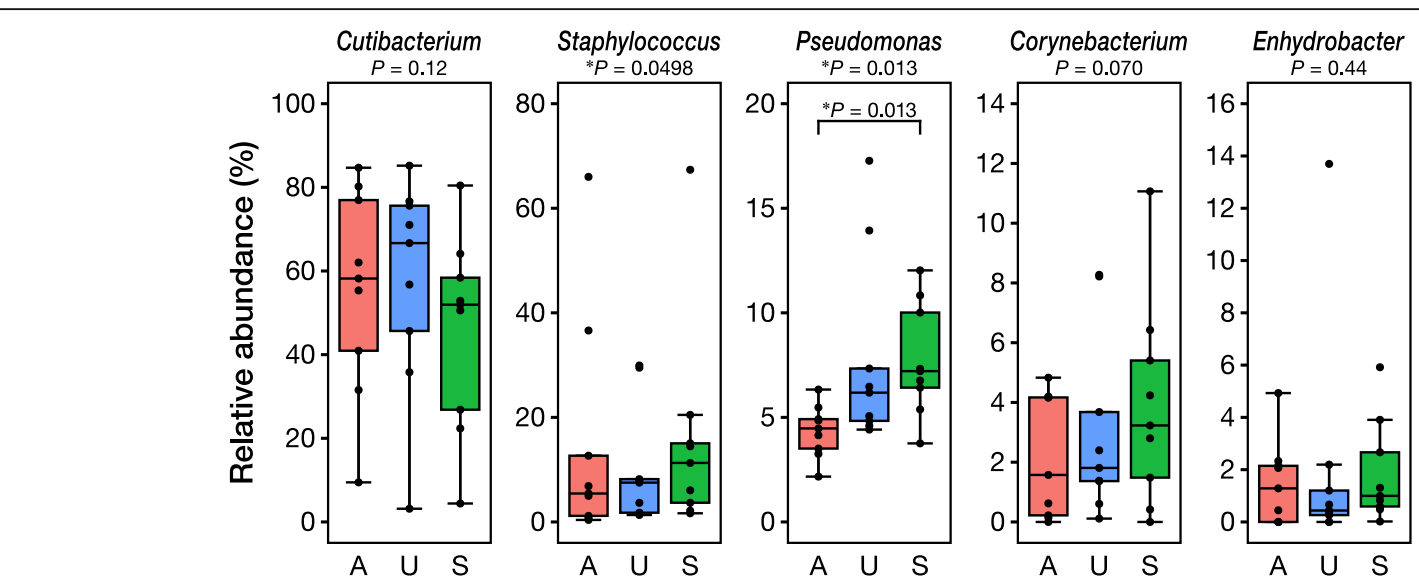

Fig. 3 Relative abundance of the top five skin microbiota collected by the three different film dressings. The $P$ values below the name of the bacteria are the results of the Friedman test, whereas the $P$ value inside each plot was calculated by the Nemenyi post-hoc pairwise test. ${ }^{*} P<0.05$. A, acrylic adhesive; $U$, urethane adhesive; $\mathrm{S}$, silicone adhesive. For details, please refer to Table 1

Table 1 Relative abundances and results of statistical evaluation of the top 20 genera obtained using tape stripping with three different types of adhesive (acrylic, urethane, and silicone) to evaluate skin microbiota

\begin{tabular}{|c|c|c|c|c|c|c|c|}
\hline \multirow[b]{2}{*}{ Genus } & \multicolumn{3}{|c|}{ Relative abundance (\%) } & \multirow{2}{*}{$\begin{array}{l}\text { Friedman } \\
\text { test } \\
P \text { value }\end{array}$} & \multicolumn{3}{|c|}{$P$ value of Nemenyi post-hoc test ${ }^{a}$} \\
\hline & Acrylic & Urethane & Silicone & & $A-U$ & $A-S$ & U-S \\
\hline Cutibacterium & $58.2(40.9-77.0)$ & $66.7(45.7-75.6)$ & $51.9(26.8-58.4)$ & 0.12 & & & \\
\hline Staphylococcus & $5.4(1.2-12.7)$ & $7.5(1.8-8.2)$ & $11.3(3.7-15.0)$ & $0.0498^{*}$ & 1.0 & 0.086 & 0.086 \\
\hline Pseudomonas & $4.5(3.5-4.9)$ & $6.2(4.8-7.3)$ & $7.2(6.4-10.0)$ & $0.013^{*}$ & 0.086 & $0.013^{*}$ & 0.76 \\
\hline Corynebacterium & $1.6(0.2-4.2)$ & $1.8(1.4-3.7)$ & $3.2(1.5-5.4)$ & 0.070 & & & \\
\hline Enhydrobacter & $1.3(0.0-2.1)$ & $0.4(0.3-1.2)$ & $1.0(0.6-2.7)$ & 0.44 & & & \\
\hline Paracoccus & $0.8(0.1-1.8)$ & $0.4(0.0-1.1)$ & $0.8(0.2-1.2)$ & 0.14 & & & \\
\hline Streptococcus & $0.0(0.0-0.4)$ & $0.3(0.0-0.6)$ & $0.8(0.3-1.5)$ & 0.069 & & & \\
\hline Lawsonella & $0.7(0.4-2.4)$ & $0.5(0.3-1.2)$ & $0.6(0.3-0.8)$ & 0.29 & & & \\
\hline Curvibacter & $0.5(0.1-0.7)$ & $0.4(0.2-1.4)$ & $0.5(0.3-0.9)$ & 0.89 & & & \\
\hline Sphingomonas & $0.6(0.2-1.0)$ & $0.3(0.3-1.7)$ & $0.5(0.0-0.9)$ & 0.92 & & & \\
\hline Gardnerella & $0.0(0.0-0.0)$ & $0.0(0.0-0.0)$ & $0.0(0.0-0.3)$ & 0.76 & & & \\
\hline Yersiniaceae & $0.4(0.4-0.5)$ & $0.6(0.3-0.9)$ & $0.6(0.3-0.8)$ & 0.46 & & & \\
\hline Brevundimonas & $0.2(0.0-0.6)$ & $0.0(0.0-0.3)$ & $0.2(0.0-0.3)$ & 0.38 & & & \\
\hline Methylobacterium-Methylorubrum & $0.5(0.3-1.0)$ & $0.1(0.0-0.8)$ & $0.3(0.0-0.4)$ & 0.24 & & & \\
\hline Bacteroides & $0.0(0.0-0.2)$ & $0.0(0.0-0.5)$ & $0.1(0.0-0.9)$ & 0.23 & & & \\
\hline Prevotella & $0.0(0.0-0.2)$ & $0.1(0.0-0.7)$ & $0.1(0.0-0.8)$ & 0.65 & & & \\
\hline Comamonas & $0.0(0.0-0.0)$ & $0.0(0.0-0.0)$ & $0.0(0.0-0.0)$ & 0.37 & & & \\
\hline Escherichia-Shigella & $0.0(0.0-0.2)$ & $0.2(0.0-0.4)$ & $0.3(0.0-1.3)$ & 0.38 & & & \\
\hline Massilia & $0.1(0.0-0.3)$ & $0.0(0.0-0.4)$ & $0.3(0.0-0.3)$ & 0.89 & & & \\
\hline Finegoldia & $0.1(0.0-0.5)$ & $0.0(0.0-1.1)$ & $0.0(0.0-0.3)$ & 0.83 & & & \\
\hline
\end{tabular}

Data are expressed as the median (interquartile range)

$A$ Acrylic adhesive, $U$ Urethane adhesive, $S$ Silicone adhesive

${ }^{*} P<0.05$

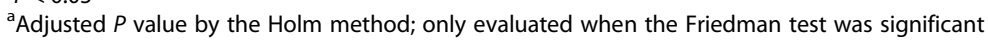



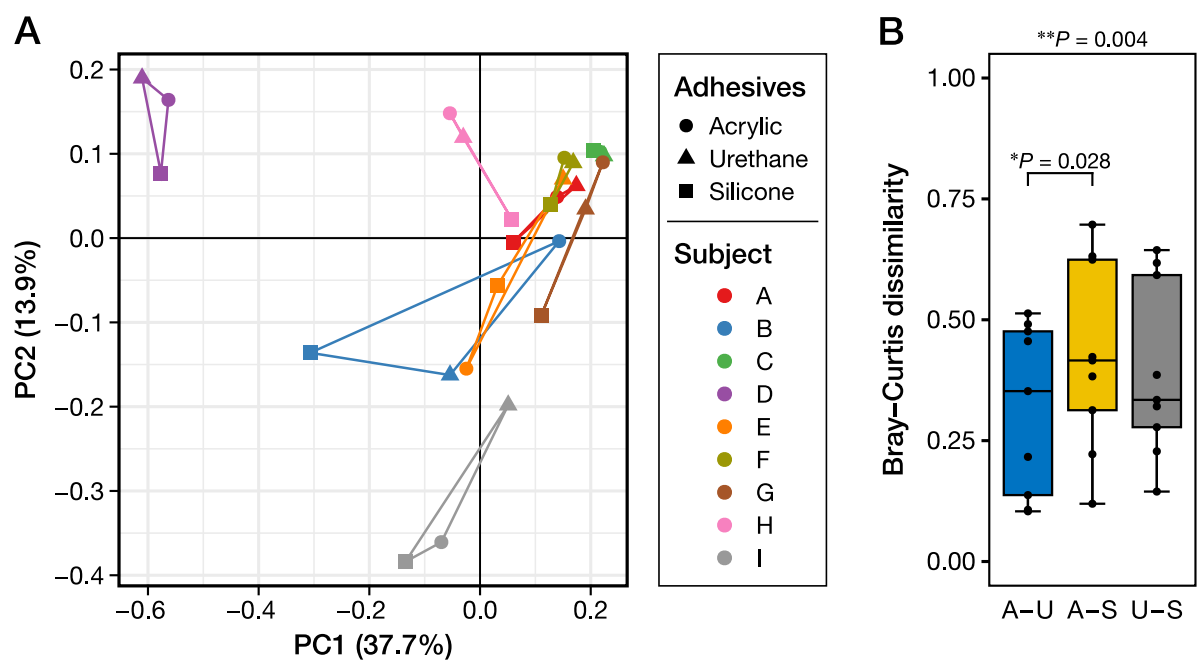

Fig. 4 Beta diversity plot based on Bray-Curtis dissimilarity (a) and pairwise comparison of Bray-Curtis dissimilarity for the three different dressings (b). a Each different color denotes each participant. The plots from the same participant are connected by lines. $\mathbf{b}$ The $P$ value outside the box is the result of the Friedman test, whereas the $P$ value inside the box is calculated by the Nemenyi $p o s t-h o c$ pairwise test. ${ }^{*} P<0.05,{ }^{* *} P<$ 0.01. PC, principal coordinate; A, acrylic adhesive; U, urethane adhesive; S, silicone adhesive. The blue box in (b), A vs. U; the yellow box, A vs. S; the gray box, U vs. S

It is crucial to collect the largest possible amount of skin microbiota (i.e., $16 \mathrm{~S}$ rRNA genes) to obtain reliable skin microbiome data [24]. To assess the differences in the yield of skin microbiota, we first determined the copy number of $16 \mathrm{~S}$ rRNA genes obtained from the three film dressings, and then we compared the results using a previously-validated dressing with acrylic adhesive as a benchmark [12]. As expected, the film dressing with acrylic adhesive yielded the highest number of $16 \mathrm{~S}$ rRNA genes, followed by the film dressing with urethane adhesive and then the film dressing with silicone adhesive (Fig. 1). Film dressings with silicone adhesive were developed to reduce the attachment force to the skin and were reported to require less removing force and to reduce skin damage and discomfort $[16,17,21,25,26]$. The significantly lower efficiency of silicone-adhesive dressings in collecting skin microbiota could be due to the reduced power of attachment to the skin. Because the use of urethane adhesive for film dressings is a relatively new concept, only a limited number of film dressings with urethane adhesive were commercially available.

Table 2 Results of PERMANOVA for beta diversity (Bray-Curtis dissimilarity)

\begin{tabular}{lll}
\hline & $\boldsymbol{P}$ value & $\boldsymbol{q}$ value $^{\mathbf{a}}$ \\
\hline Overall & 0.77 & - \\
Acrylic - Urethane & 0.96 & 0.96 \\
Acrylic - Silicone & 0.41 & 0.91 \\
Urethane - Silicone & 0.61 & 0.91 \\
\hline
\end{tabular}

${ }^{a}$ Adjusted $P$ value by means of the Benjamini-Hochberg false discovery rate control
The urethane adhesive product that we used employed a urethane gel as an adhesive with a view to high adhesiveness and low irritation [20]. The number of 16S rRNA genes obtained by the urethane adhesive fell between the amount obtained by the acrylic and silicone adhesives and was not significantly less than the acrylic adhesive (Fig. 1). Bender et al. reported that microbial results could be biased if the input copy number of $16 \mathrm{~S}$ rRNA genes was low and suggested that at least 100 copies were required for NGS analysis [24]. From our study, when assuming that $1 \mu \mathrm{L}$ of the DNA solution is used as a template for NGS, no dressings with acrylic adhesive, $1 / 9$ dressings with urethane adhesive, and 5/9 dressings with silicone adhesive did not reach the threshold (100 copies, [24]) (Fig. 1). Therefore, it is expected that dressings with acrylic or urethane adhesive would be the first choice for amplicon-based skin microbiome studies, prioritized according to yield or safety, respectively.

Next, we assessed the microbiome results obtained from the three film dressings. All three film dressings had the same top five skin microbiota (Figs. 2 and 3, and Table 1), namely, Cutibacterium spp., Staphylococcus spp., Pseudomonas spp., Corynebacterium spp., and Enhydrobacter spp. However, the dressing with silicone adhesive showed significantly different results in the relative abundance of Pseudomonas spp., and less, albeit not significant, Cutibacterium spp. (Figs. 2 and 3, and Table 1). The ASV-level analysis also showed simiar results in which the dressing with silicone adhesive yielded different results in comparison with the dressing with acrylic or urethane adhesive (Additional file 2: Table S1). 

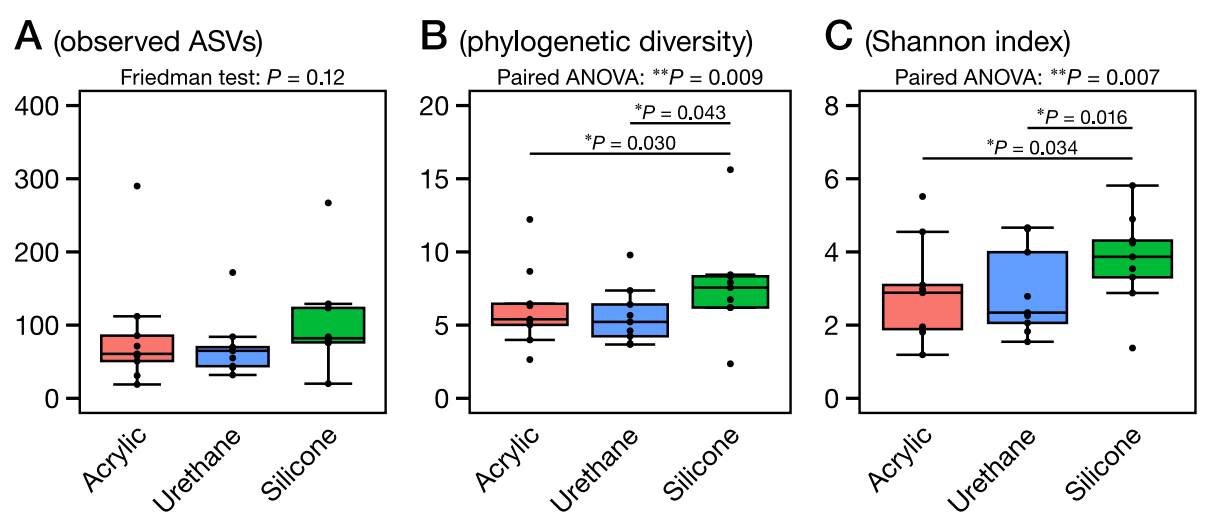

Fig. 5 Alpha diversity indices: the number of observed amplicon sequence variants (ASVs) (a), phylogenetic diversity (b), and Shannon index (c). The $P$ values outside the box are the results from the Friedman test or one-way analysis of variance (ANOVA) of repeated measures, whereas the $P$ values inside each box were calculated by the Nemenyi post-hoc pairwise test or Welch's pairwise $t$-test with Holm adjustment

Additionally, the Bray-Curtis dissimilarity was significantly higher between the acrylic- and silicone-adhesive film dressings than between the acrylic and urethane film dressings (Fig. 4b), although there were no significant differences in the principal coordinate analysis distribution as a whole (Fig. 4a). Finally, the dressing with silicone adhesive showed significantly higher alpha diversity indices (phylogenetic diversity and Shannon index) than the other two film dressings (Fig. 5). Thus, we presumed that the film dressing with silicone adhesive leads to subtly different results compared with the other two adhesive film dressings. This can be explained by differences in the amount of template and PCR cycles. In this study, for the samples with a smaller 16S rRNA gene copy number (especially those derived from the film dressing with silicone adhesive), the number of PCR cycles was increased by $1-4$ cycles to achieve a sufficient amount of amplified product (Additional file 3: Table S2). Brooks et al. reported that the number of PCR cycles could slightly affect the results of the 16S rRNA gene sequence data [27]. Given the situation where samples with an insufficient amount of template (Fig. 1) were amplified to achieve sufficient product for NGS analysis, it is possible that the differences in the cycling conditions might have affected the results. However, Sze and Schloss mentioned that KAPA HiFi HS enzyme, which was used in this study, gave the most stable results, irrespective of a higher number of PCR cycles, among the PCR enzymes tested [28]. Furthermore, the use of KAPA HiFi HS enzyme was reported to lead to a lower Shannon index when the PCR cycles were increased [28], which contradicts the findings of this study. Therefore, there may be another explanation for why the dressing with silicone adhesive yielded slightly different results.

Our study had several limitations. First, we only tested one position (back skin), as in our previous study [12], and a different position might give different results, as the skin microbiome is diverse across body sites [29]. Second, we did not test the three film dressings on fragile skin (e.g., older people), so we could not directly prove the applicability and safety of the film dressing with a urethane adhesive to those who have fragile skin. Nonetheless, we have already utilized the urethaneadhesive film dressing for skin microbiome study on older people with fragile skin (age range, 71-100 years old), and there were no adverse events [23]; therefore, the dressing with urethane adhesive can be considered safe as such for very old people. Third, the number of participants might be small for comparison. In this study, we have recruited nine participants as with our previous study [12] and the studies of other groups [22, 30-33]. The smaller number of samples leads to less statistical power for significance tests; therefore, the non-significant difference in the microbial composition between acrylic and urethane dressings found in this study could also be explained by the small number of samples. However, at least, silicone adhesive would not be recommended for skin microbiome studies, as it showed significantly less microbial yield and different microbial composition even under small number of samples. That being said, it would be advised that the sampling method should be fixed (i.e., acrylic or urethane) throughout a single study. Finally, more detailed comparison by whole genome sequencing with speciesor strain-level analysis would give different results when compared to amplicon-based metagenomics. Further studies with whole genome metagenomics are warranted.

\section{Conclusion}

This study showed that film dressings with acrylic or urethane adhesive should be chosen when used to obtain skin samples for amplicon-based microbiome studies. 
The choice of acrylic or urethane adhesive can be based on what requires prioritization: acrylic adhesive for the yield of skin microbiota or urethane adhesive for skin safety, such as when sampling from older people. A film dressing with silicone adhesive was unsuitable for the purpose of skin microbiome studies, yielding measurably less skin microbiota and subsequent biased results.

\section{Methods}

\section{Ethical approval}

This study was approved by the Medical Ethics Committee of Kanazawa University, Kanazawa, Japan (approval number 803). The researchers followed the Declaration of Helsinki and the Microorganism Safety Management Regulations of Kanazawa University. Bacterial samples were processed in a level 2 biosafety laboratory.

\section{Participants}

We recruited nine healthy young participants (four females, age 21-22 years old; five males, age 20-21 years old). Written informed consent was obtained from all participants prior to inclusion. No participants had skin disorders or any systemic diseases. Additionally, none of the participants reported the use of topical or systemic antibiotics within 2 weeks prior to study inclusion. The participants were requested to not use creams or lotions on the site of sample collection (back skin) within $24 \mathrm{~h}$ of the day of sampling and to not take a bath or shower after midnight on the day before sampling. All the participant were confirmed to have complied with the requests.

\section{Film dressings}

We selected three types of film dressings with different adhesives for tape stripping: (1) a film dressing with acrylic adhesive Tegaderm $^{\text {tox }}$ Transparent Film Dressing, $1622 \mathrm{~W} ; 3$ M Company, MN, USA), (2) a film dressing with urethane adhesive (Cathereeplus ${ }^{\mathrm{Tm}}$, CPS0405; Nichiban Co., Ltd., Tokyo, Japan), and (3) a film dressing with silicone adhesive (Mepitel Film, 296,170; Mölnlycke Health Care, Gothenburg, Sweden). The size of each film dressing was $\sim 20 \mathrm{~cm}^{2}$ (acrylic, $4.4 \times 4.4 \mathrm{~cm}, 19.4 \mathrm{~cm}^{2}$; urethane, $4 \times 5 \mathrm{~cm}, 20 \mathrm{~cm}^{2}$; and silicone, $6 \times 7 \mathrm{~cm}$, which was cut in half to $6 \times 3.5 \mathrm{~cm}, 21 \mathrm{~cm}^{2}$ ).

\section{Tape stripping}

Tape stripping was performed with the three different film dressings. First, the participant's back was inspected to check that there were no apparent skin diseases (e.g., rash, wound, or sores). The position (back skin) was chosen as in our previous study [12]. Then, three neighboring areas were designated, and each film dressing was applied randomly to each designated area (Additional file 4: Figure S2). All the film dressings were pressed once using a sterile roller (MSR0001; Bio-Rad Laboratories, Inc., CA, USA) with constant pressure to ensure attachment to the skin. After $1 \mathrm{~min}$, the film dressings were removed from the skin using sterile round-tip tweezers and placed in separate sterile plastic containers. The film dressings were stored at $-80^{\circ} \mathrm{C}$ until DNA extraction.

\section{DNA extraction}

Bacterial DNA was extracted from the film dressings as described previously [12, 34]. Briefly, using a safety cabinet, each film dressing was minced by sterile scissors, followed by chemical (1.2\% Triton-X 100) and enzymatical (lysozyme and lysostaphin) disruption of the bacterial cell walls. The resultant solution was then cleaned-up using a QIAamp DNA Mini Kit (QIAGEN, Venlo, The Netherlands) according to the manufacturer's instructions. Each extracted bacterial DNA sample was recovered in $100 \mu \mathrm{L}$ of $\mathrm{AE}$ buffer $(10 \mathrm{mM}$ Tris- $\mathrm{HCl}, 0.5 \mathrm{mM}$ ethylenediaminetetraacetic acid, $\mathrm{pH}$ 9.0). The DNA solution was stored at $-20^{\circ} \mathrm{C}$ until analysis.

\section{Library preparation for NGS}

Quantitative polymerase chain reaction (qPCR) for $16 \mathrm{~S}$ rRNA gene quantification was performed on the extracted bacterial DNA, as described elsewhere [12], with THUNDERBIRD ${ }^{\circ}$ SYBR $^{\circ}$ qPCR Mix (Toyobo Co., Ltd., Osaka, Japan) and 16S rRNA primers (forward: $5^{\prime}$-ACTGAGAYACGGYCCA-3' and reverse: 5'-CTGCTGGC ACGDAGTTAGCC-3') on an AriaMX Real-Time PCR System (Agilent Technologies, Inc., CA, USA). Then, equal concentrations of the resultant amplified DNA solutions containing the 16S rRNA gene were used to amplify the V3-V4 hypervariable region, to better capture the microbial composition of the skin [30], with KAPA HiFi HS ReadyMix (F. Hoffmann-La Roche, Ltd., Basel, Switzerland) and primers with MiSeq-compatible overhang sequences (forward: 5'-TCGTCGGCAG CGTCAGATGTGTATAAGAGACAGCCTACGGGNG GCWGCAG-3' and reverse: 5'-GTCTCGTGGGCTCG GAGATGTGTATAAGAGACAGGACTACHVGGGTA TCTAAKCC-3'). After cleaning up the polymerase chain reaction (PCR) products using AMPure XP magnetic beads (Beckman Coulter Inc., CA, USA), indexing PCR was performed for all samples using a Nextera XT Index Kit v2 (Illumina Inc., CA, USA) followed by AMPure XP cleaning. The concentration of each product was measured by means of a Qubit dsDNA HS Assay Kit (Thermo Fisher Scientific, MA, USA), and equimolar amounts of the products were mixed to create the final library solution. 


\section{NGS}

The library solution was spiked with $15 \%$ PhiX Control v3 (Illumina Inc.) and loaded in a flow cell of a MiSeq Reagent Kit v3 (600 cycles; Illumina Inc.) on a MiSeq system (Illumina Inc.). NGS was performed according to the manufacturer's instructions.

\section{Bioinformatics analyses}

All the microbiome analyses were performed using QIIME 2 software (version 2019.10) [35] and the SILVA rRNA database (release 138) [36]. The raw fastq sequence files were first filtered, and chimeras were eliminated using the DADA2 plugin [37] with default settings. The taxonomic assignment was then performed using the classify-sklearn method with the trained classifier created by the SILVA database and the fit-classifiernaive-bayes method in the q2-feature-classifier plugin [38]. Alpha diversity indices (the number of observed ASVs, Faith's phylogenetic diversity, and Shannon index) and beta diversity (based on Bray-Curtis dissimilarity [39]) were calculated by the core-metrics-phylogenetic pipeline of the q2-diversity plugin with rarefaction at a depth of 12,627 sequences, the minimum read number among all the samples. The rarefaction curves of all samples are shown in Additional file 5: Figure S3.

\section{Statistics}

The data were expressed as averages for the cumulative bar charts of relative bacterial abundance or as medians with interquartile range (IQR) and median $-1.5 \times$ IQR to median + 1.5 $\times$ IQR whiskers for the other parameters. The 16S rRNA gene copy number, relative abundances of representative bacterial genera, alpha diversity indices, and dissimilarity index for beta diversity among the three different film dressings were evaluated by the Friedman test followed by the Nemenyi post-hoc multiple comparison test using the PMCMRplus package [40] or one-way analysis of variance (ANOVA) of repeated measures followed by pairwise Welch's $t$-test with $P$ value adjustment by the Holm method using the car package [41], according to data distribution. The difference in beta diversity among the three film dressings was statistically evaluated by PERMANOVA tests followed by $P$ value adjustment with Benjamini-Hochberg false discovery rate (FDR) control using betagroup-significance visualizer of QIIME 2. A $P$ value or $q$ value (adjusted $P$ value by FDR control) $<0.05$ was considered statistically significant. For statistical evaluation, $\mathrm{R}$ software (version 3.6.2) [42] and the QIIME 2 built-in analyzer were used.

\section{Data availability}

All raw fastq sequences are available from the DNA Data Bank of Japan (accession no. DRA009701).

\section{Supplementary Information}

The online version contains supplementary material available at https://doi. org/10.1186/s12866-021-02122-4.

Additional file 1: Figure S1. Relative abundance of the top 20 genera of skin microbiota for each participant. A, acrylic adhesive; U, urethane adhesive; S, silicone adhesive.

Additional file 2: Table S1. Amplicon sequence variant (ASV)-level comparison between three different adhesives. ASVs that shared more than $0.1 \%$ on average are listed.

Additional file 3: Table S2. Cycle numbers in the first PCR.

Additional file 4: Figure S2. Position of sample collection.

Additional file 5: Figure S3. Rarefaction curves for each alpha diversity index. ASV, amplicon sequence variants. Vertical bars denote 12,627 reads, the minimum read number among all the samples.

\section{Acknowledgments}

The authors would like to thank the participants of this study. The authors would like to acknowledge Ms. Emi Matsubara and Ms. Risa Okamoto for their experimental assistance.

\section{Authors' contributions}

$\mathrm{SO}$ and JS conceived the study, KO1, KS, NT, and $\mathrm{KO} 2$ acquired data, KO1 and $\mathrm{KO} 2$ analyzed data, $\mathrm{KO} 1$ wrote the original draft, and all authors approved the final manuscript.

\section{Funding}

This study was supported in part by the Ministry of Education, Culture, Sports, Science and Technology KAKENHI Grant No. $17 \mathrm{H} 04428$ to SO, and "Initiative for the implementation of the diversity research environment (Collaboration type)." to JS. None of the funding bodies were involved in the design of the study and collection, analysis, and interpretation of data and in writing the manuscript.

\section{Availability of data and materials}

The datasets generated and/or analysed during the current study are available in the DNA Data Bank of Japan (accession no. DRA009701). https:// ddbj.nig.ac.jp/DRASearch/submission?acc=DRA009701

\section{Ethics approval and consent to participate}

This study was approved by the Medical Ethics Committee of Kanazawa University, Kanazawa, Japan (approval number 803). Written informed consent was obtained from all participants prior to inclusion.

\section{Consent for publication}

Not applicable.

\section{Competing interests}

The authors declare that they have no competing interests.

\section{Author details}

${ }^{1}$ Al Hospital/Macro Signal Dynamics Research and Development Center, Institute of Medical, Pharmaceutical and Health Sciences, Kanazawa University, 5-11-80 Kodatsuno, Kanazawa, Ishikawa 9200942, Japan. ${ }^{2}$ Department of Nursing, Faculty of Health Sciences, Institute of Medical, Pharmaceutical and Health Sciences, Kanazawa University, 5-11-80 Kodatsuno, Kanazawa, Ishikawa 9200942, Japan. ${ }^{3}$ Department of Clinical Laboratory Science, Faculty of Health Sciences, Institute of Medical, Pharmaceutical and Health Sciences, Kanazawa University, 5-11-80 Kodatsuno, Kanazawa, Ishikawa 9200942, Japan. ${ }^{4}$ Advanced Health Care Science Research Unit, Institute for Frontier Science Initiative, Kanazawa University, 5-11-80 Kodatsuno, Kanazawa, Ishikawa 9200942, Japan.

Received: 25 August 2020 Accepted: 9 February 2021

Published online: 18 February 2021

\section{References}

1. Rawlings AV, Harding CR. Moisturization and skin barrier function. Dermatol Ther. 2004;17(Suppl 1):43-8. https://doi.org/10.1111/j.1396-0296.2004.04s1005.x. 
2. Matejuk A. Skin Immunity. Arch Immunol Ther Exp. 2018;66(1):45-54. https:// doi.org/10.1007/s00005-017-0477-3.

3. Choi EH. Aging of the skin barrier. Clin Dermatol. 2019;37(4):336-45. https:// doi.org/10.1016/j.clindermatol.2019.04.009.

4. Chiller K, Selkin BA, Murakawa GJ. Skin microflora and bacterial infections of the skin. J Investig Dermatol Symp Proc. 2001;6(3):170-4. https://doi.org/10.1 046/j.0022-202x.2001.00043.x.

5. Kosiewicz MM, Zirnheld AL, Alard P. Tuning of skin immunity by skin commensal bacteria. Immunotherapy. 2013;5(1):23-5. https://doi.org/10.2217/imt.12.140.

6. Chang HW, Yan D, Singh R, Liu J, Lu X, Ucmak D, et al. Alteration of the cutaneous microbiome in psoriasis and potential role in Th17 polarization. Microbiome. 2018:6(1):154. https://doi.org/10.1186/s40168-018-0533-1.

7. Cinque B, Palumbo P, La Torre C, Melchiorre E, Corridoni D, Miconi G, et al. Probiotics in Aging Skin. In: Farage MA, Miller KW, Maibach HI, editors. Textbook of Aging Skin. Berlin: Springer Berlin Heidelberg; 2017. p. 1315-27.

8. Human Microbiome Project Consortium. Structure, function and diversity of the healthy human microbiome. Nature. 2012;486(7402):207-14. https://doi. org/10.1038/nature11234.

9. Human Microbiome Project Consortium. A framework for human microbiome research. Nature. 2012;486(7402):215-21. https:/doi.org/10.1038/nature11209.

10. Kong HH, Andersson B, Clavel T, Common JE, Jackson SA, Olson ND, et al. Performing skin microbiome research: a method to the madness. J Invest Dermatol. 2017;137(3):561-8. https://doi.org/10.1016/j.jid.2016.10.033.

11. Chng KR, Tay AS, Li C, Ng AH, Wang J, Suri BK, et al. Whole metagenome profiling reveals skin microbiome-dependent susceptibility to atopic dermatitis flare. Nat Microbiol. 2016;1(9):16106. https://doi.org/10.1038/ nmicrobiol.2016.106.

12. Ogai K, Nagase S, Mukai K, luchi T, Mori Y, Matsue M, et al. A comparison of techniques for collecting skin microbiome samples: swabbing versus tapestripping. Front Microbiol. 2018;9:2362. https://doi.org/10.3389/fmicb.2018.02362.

13. Tanaka A, Cho O, Saito C, Saito M, Tsuboi R, Sugita T. Comprehensive pyrosequencing analysis of the bacterial microbiota of the skin of patients with seborrheic dermatitis. Microbiol Immunol. 2016;60(8):521-6. https://doi. org/10.1111/1348-0421.12398.

14. Zeeuwen PL, Boekhorst J, van den Bogaard EH, de Koning HD, van de Kerkhof PM, Saulnier DM, et al. Microbiome dynamics of human epidermis following skin barrier disruption. Genome Biol. 2012;13(11):R101. https://doi. org/10.1186/gb-2012-13-11-r101.

15. Lange-Asschenfeldt B, Marenbach D, Lang C, Patzelt A, Ulrich M, Maltusch A, et al. Distribution of bacteria in the epidermal layers and hair follicles of the human skin Skin Pharmacol Physiol. 2011;24(6):305-11. https:/doi.org/10.1159/000328728.

16. White R. Evidence for atraumatic soft silicone dressing use. Wounds-UK. 2005;1(3):104-9.

17. Morris C, Emsley P, Marland E, Meuleneire F, White R. Use of wound dressings with soft silicone adhesive technology. Paediatr Nurs. 2009;21(3): 38-43. https://doi.org/10.7748/paed2009.04.21.3.38.c7037.

18. Konya C, Sanada H, Sugama J, Okuwa M, Kamatani Y, Nakagami G, et al. Skin injuries caused by medical adhesive tape in older people and associated factors. J Clin Nurs. 2010;19(9-10):1236-42. https://doi.org/1 0.1111/j.1365-2702.2009.03168.x.

19. Zulkowski K. Understanding moisture-associated skin damage, medical adhesive-related skin injuries, and skin tears. Adv Skin Wound Care. 2017; 30(8):372-81. https://doi.org/10.1097/01.ASW.0000521048.64537.6e.

20. Nichiban Co. Ltd. CATHEREEPLUS product information website. https//www.nichiba n.com/products/medical/dressing/cathereeplus.html Accessed 27 Feb 2020

21. White R. A multinational survey of the assessment of pain when removing dressings. Wounds-UK. 2008:4(1):14-22.

22. Grice EA, Kong HH, Renaud G, Young AC, Program NCS, Bouffard GG, et al. A diversity profile of the human skin microbiota. Genome Res. 2008;18(7): 1043-50. https://doi.org/10.1101/gr.075549.107.

23. Nagase $S$, Ogai K, Urai T, Shibata K, Matsubara E, Mukai K, et al. Distinct skin microbiome and skin physiological functions between bedridden older patients and healthy people: a single-center study in Japan. Front Med (Lausanne). 2020;7:101. https://doi.org/10.3389/fmed.2020.00101.

24. Bender JM, Li F, Adisetiyo H, Lee D, Zabih S, Hung L, et al. Quantification of variation and the impact of biomass in targeted $16 \mathrm{~S}$ rRNA gene sequencing studies. Microbiome. 2018;6(1):155. https://doi. org/10.1186/s40168-018-0543-z.

25. Dykes PJ, Heggie R. The link between the peel force of adhesive dressings and subjective discomfort in volunteer subjects. J Wound Care. 2003;12(7): 260-2. https://doi.org/10.12968/jowc.2003.12.7.26567.
26. Dykes PJ, Heggie R, Hill SA. Effects of adhesive dressings on the stratum corneum of the skin. J Wound Care. 2001;10(2):7-10. https://doi.org/10.12 968/jowc.2001.10.2.26054.

27. Brooks JP, Edwards DJ, Harwich MD Jr, Rivera MC, Fettweis JM, Serrano MG, et al. The truth about metagenomics: quantifying and counteracting bias in 165 rRNA studies. BMC Microbiol. 2015;15:66. https://doi.org/10.1186/s12866-015-0351-6.

28. Sze MA, Schloss PD. The Impact of DNA Polymerase and Number of Rounds of Amplification in PCR on 165 rRNA Gene Sequence Data. mSphere. 2019; 4(3). https://doi.org/10.1128/mSphere.00163-19.

29. Grice EA, Segre JA. The skin microbiome. Nat Rev Microbiol. 2011;9(4):24453. https://doi.org/10.1038/nrmicro2537.

30. Castelino M, Eyre S, Moat J, Fox G, Martin P, Ho P, et al. Optimisation of methods for bacterial skin microbiome investigation: primer selection and comparison of the 454 versus MiSeq platform. BMC Microbiol. 2017;17(1):23. https://doi.org/10.1186/s12866-017-0927-4.

31. Bassiouni A, Cleland EJ, Psaltis AJ, Vreugde S, Wormald PJ. Sinonasal microbiome sampling: a comparison of techniques. PLoS One. 2015;10(4): e0123216. https://doi.org/10.1371/journal.pone.0123216.

32. Klymiuk I, Bambach I, Patra V, Trajanoski S, Wolf P. 16 S based microbiome analysis from healthy Subjects' skin swabs stored for different storage periods reveal phylum to genus level changes. Front Microbiol. 2016;7:2012. https://doi.org/10.3389/fmicb.2016.02012.

33. Bjerre RD, Hugerth LW, Boulund F, Seifert M, Johansen JD, Engstrand L. Effects of sampling strategy and DNA extraction on human skin microbiome investigations. Sci Rep. 2019;9(1):17287. https://doi.org/10.1038/ s41598-019-53599-z.

34. Yuan S, Cohen DB, Ravel J, Abdo Z, Forney LJ. Evaluation of methods for the extraction and purification of DNA from the human microbiome. PLoS One. 2012;7(3):e33865. https://doi.org/10.1371/journal.pone.0033865.

35. Bolyen E, Rideout JR, Dillon MR, Bokulich NA, Abnet CC, Al-Ghalith GA, et al. Reproducible, interactive, scalable and extensible microbiome data science using QIIME 2. Nat Biotechnol. 2019;37(8):852-7. https//doi.org/10.1038/s41587-019-0209-9.

36. Quast C, Pruesse E, Yilmaz P, Gerken J, Schweer T, Yarza P, et al. The SILVA ribosomal RNA gene database project: improved data processing and webbased tools. Nucleic Acids Res. 2013;41(Database issue):D590-6. https://doi. org/10.1093/nar/gks1219.

37. Callahan BJ, McMurdie PJ, Rosen MJ, Han AW, Johnson AJ, Holmes SP. DADA2: high-resolution sample inference from Illumina amplicon data. Nat Methods. 2016;13(7):581-3. https://doi.org/10.1038/nmeth.3869.

38. Bokulich NA, Kaehler BD, Rideout JR, Dillon M, Bolyen E, Knight R, et al. Optimizing taxonomic classification of marker-gene amplicon sequences with QIIME 2's q2-feature-classifier plugin. Microbiome. 2018;6(1):90. https:// doi.org/10.1186/s40168-018-0470-z.

39. Faith DP, Minchin PR, Belbin L. Compositional dissimilarity as a robust measure of ecological distance. Vegetatio. 1987;69(1):57-68. https://doi. org/10.1007/BF00038687.

40. Pohlert T. PMCMRplus: Calculate Pairwise Multiple Comparisons of Mean Rank Sums Extended. https://cran.r-project.org/web/packages/PMCMRplus/ index.html Accessed 29 Feb 2020.

41. Fox J, Weisberg S. An R companion to applied regression. 3rd ed. Thousand Oaks: SAGE Publications; 2018.

42. R Core Team. R: A Language and Environment for Statistical Computing. https://www.R-project.org/ Accessed 29 Feb 2020.

\section{Publisher's Note}

Springer Nature remains neutral with regard to jurisdictional claims in published maps and institutional affiliations.

\section{Ready to submit your research? Choose BMC and benefit from:}

- fast, convenient online submission

- thorough peer review by experienced researchers in your field

- rapid publication on acceptance

- support for research data, including large and complex data types

- gold Open Access which fosters wider collaboration and increased citations

- maximum visibility for your research: over $100 \mathrm{M}$ website views per year

At $\mathrm{BMC}$, research is always in progress.

Learn more biomedcentral.com/submissions 